Case Study

\title{
Autoimmune Hepatitis and Primary Sclerosing Cholangitis: An Overlap Syndrome Resistant to Azathioprine and Intolerant to Tacrolimus with Sepsis Mimicking Flare UP-A Clinical Dilemma
}

\author{
Sharon TMathews', Deepika Poonia ${ }^{2}$, Naresh Kumar $^{3}, \underline{\text { M Bhavana }}^{4}, \underline{\text { RS Ahlawat }}^{5}$, \\ Harpreet Singh ${ }^{6}$, Suresh Kumar ${ }^{7}$, Sandeep Garg ${ }^{8}$
}

${ }^{1}$ Assistant Professor, ${ }^{4}$ Postgraguate, ${ }^{5}$ Director Professor, ${ }^{6}$ Associate Professor, ${ }^{7}$ Director Professor \& Head, ${ }^{8}$ Professor, Department of Medicine, Maulana Azad Medical College, New Delhi, India.

${ }^{2}$ Assistant Professor, Department of Anatomy, Maulana Azad Medical College, New Delhi, India.

${ }^{3}$ Professor of Medicine and Head, Pulmonary Medicine, Maulana Azad Medical College, New Delhi, India.

DOI: https://doi.org/10.24321/2349.7181.202006

I $\quad \mathbf{N} \quad \mathbf{F} \mathbf{O}$

Corresponding Author:

Sharon T Mathews, Department of Medicine, Maulana Azad Medical College, New Delhi, India.

E-mail Id:

sharontmathews@gmail.com

Orcid Id:

https://orcid.org/0000-0003-2257-9909

How to cite this article:

Mathews ST, Poonia D, Kumar N, Bhavana M, Ahlawat RS, Singh $\mathrm{H}$ et al. Autoimmune Hepatitis and Primary Sclerosing Cholangitis: An Overlap Syndrome Resistant to Azathioprine and Intolerant to Tacrolimus with Sepsis Mimicking Flare up-A Clinical Dilemma. J Adv Res Med 2020; 7(2): 11-14.

Date of Submission: 2020-08-01

Date of Acceptance: 2020-08-12

\section{$\begin{array}{llllllll}\text { A } & \text { B } & \mathbf{S} & \mathbf{T} & \mathbf{R} & \mathbf{A} & \mathbf{C} & \mathbf{T}\end{array}$}

Patients with autoimmune liver disease in a frequency of $7 \%-18 \%$ fit into the diagnostic criteria for more than one condition. Up to $12.5 \%$ of Autoimmune Hepatitis (AIH) and Primary Sclerosing Cholangitis (PSC) cohorts have a label of AIH/PSC overlap. There can be an unpredictable interval of many years between the diagnosis of the two conditions. There are scoring systems but no established criteria for the diagnosis of AIH/PSC overlap. Therefore, a diagnosis based on combination of biochemistry, autoantibody profile, cholangiogram, and liver histologyis made. Histopathology staging of AIH/PSC overlap influences therapeutic options and prognosis. There is beneficial role of immunosuppression, even though there is a higher relapse rate and evidence of progressive liver disease despite immunosuppression in some cases. Liver related outcomes in this overlap are better than PSC alone but are poorer than AlH. We herein report a rare presentation of an overlap syndrome with AIH and PSC in a patient in whom, treatment with first line therapy showed incomplete response and the salvage therapy was not tolerated, presenting with Acute Kidney Injury (AKI) and a clinical picture of sepsis resembling AlH flare.

Keywords: Autoimmune Hepatitis, Primary Sclerosis Cholangitis, Overlap Syndrome, Azathioprine, Tacrolimus, Mycophenolate 


\section{Introduction}

The Autoimmune Liver Diseases (AILD) have been divided as either Autoimmune Hepatitis (AlH), Primary Sclerosing Cholangitis (PSC), or Primary Biliary Cholangitis (PBC). They are a heterogeneous group of conditions showing differing pathogenesis, patterns of hepatic injury, and clinical outcomes. ${ }^{1}$ Despite this, AIH, PSC, and PBC are can be grouped together as AILD because of similarities in clinical presentation, immunological markers, and treatment options. A group of patients with AILD share common features relating to different subtypes of AILD; these have been labelled as 'overlap syndromes' or 'variant syndromes.' In this evolving field, management principles even among experts remain heterogeneous. These variations in therapeutic approaches are the result of small retrospective studies, poor understanding of disease associated immunologic mechanisms, and wide knowledge gaps in disease pathogenesis. Unfortunately, not all patients with AlH will have favourable response to first-line regimens because of medication intolerance (10\%), ${ }^{2}$ incomplete response $(15 \%),{ }^{3}$ and treatment failure $(9 \%){ }^{4}$

\section{Case Report}

A 21-year-old male and a diagnosed case of overlap syndrome of AlH and PSC 5 years ago, presented with chief complaints of yellowish discoloration of eyes, pruritis, malaise and easy fatiguability for 1 month and fever, multiple episodes of vomiting, burning micturition and decreased urinary output for 10 days. There was no history of arthralgia, abdominal pain, clay coloured stools, bleeding episodes, altered sensorium, altered bladder and bowel habits or weight loss. Five years ago, when the patient was diagnosed with AIH and PSC overlap syndrome, the patient presented with jaundice and on investigating further, he had an AST/ALT levels $>5$ times the ULN, an ALP level of $>2000$ U/L, anti-smooth muscle antibodies were positive $(2+)$, cANCA was positive and the lgG levels $>1.1$ times the ULN. The rest of the autoimmune profile was negative. Ultrasound abdomen revealed hepatosplenomegaly, liver biopsy showed features of chronic inflammation with focal interface hepatitis, hepatocyte resetting, duct injury and duct loss suggestive of AlH with cholangiopathy. Initial MRCP was suggestive of irregular narrowing and dilatation of the intrahepatic biliary radicles and common bile duct giving a beaded appearance suggestive of PSC. In view of the diagnosis of AIH and PSC overlap syndrome, the patient was started on prednisolone which was tapered over a duration of 4 weeks with a plan to use azathioprine for maintenance therapy along with ursodeoxycholic acid. During follow up, prednisolone was changed to budesonide in view of lesser systemic toxicity. Patient initially responded to steroids and by the end of one month, the AST/ALT decreased to 3 times the ULN and the ALP reduced to $300 \mathrm{U} / \mathrm{L}$. However, despite continuing steroid for 3 months, the AST/ALT levels remained higher than 3 times the ULN and the ALP increased again to $800 \mathrm{U} / \mathrm{L}$, at which point of time azathioprine was also initiated. Patient was followed up for 3 years during which the AST/ALT values remained more than 3 times the ULN and the ALP values remained in the range of 200-250U/L. In view of persistently high levels of transaminases, the dose of azathioprine was increased, despite which the LFT's did not improve and subsequently ALP also started to rise again. Then liver biopsy was repeated which revealed the same findings as were present 3 years ago. Hence patient was considered azathioprine resistant and was started on tacrolimus along with continuation of ursodeoxycholic acid. This continued for a period of 6 months after which the patient started developing the above-mentioned complaints.

This time the investigations showed a normal AST/ALT levels with an increased bilirubin $(>21 \mathrm{mg} / \mathrm{dl})$ and ALP levels (>300U/L) suggesting a cholestatic pattern and KFT's were suggestive of acute Kidney Injury (AKI) with a blood urea of $130 \mathrm{mg} / \mathrm{dl}$ and serum creatinine reaching $6.8 \mathrm{mg} / \mathrm{dl}$, which gradually worsened in the subsequent days. Urine routine microscopy revealed significant number of pus cells. The MRCP repeated this time showed no evidence of any periportal fibrotic changes. Serum tacrolimus levels were slightly elevated than the ULN. Hence after ruling out other possible differentials, a diagnosis of tacrolimus induced nephrotoxicity superimposed with acute urinary tract infection was made. Hence, tacrolimus was tapered and stopped and steroids were restarted along with ursodeoxycholic acid. The UTI was treated with antibiotics after drug sensitivity testing, after which the cholestatic jaundice improved indicating that the cholestasis developed was more likely to be a complication of the ongoing sepsis rather than a flare up of the underlying overlap syndrome. Patient's condition subsequently improved with the bilirubin levels reducing to a level of $3 \mathrm{mg} / \mathrm{dl}$. In view of azathioprine resistance and intolerance to tacrolimus, patient was started on Mycophenolate Mofetil (MMF) as maintenance therapy along with steroids. Later, steroids were tapered. Patient was doing well thereafter but has been counselled for a liver transplant.

\section{Discussion}

AlH/PSC overlap is usually seen in young adults, in whom there a characteristic clinical, biochemical, immunological, and histological picture suggestive of AlH with co-existing classical cholangiogram of PSC (Table 1). ${ }^{5,6}$ 
Table I.Diagnostic Criteria of AlH+PSC Overlap Syndrome

\begin{tabular}{|l|l|}
\hline & \\
\hline \multirow{3}{*}{$\begin{array}{l}\text { Clinical and laboratory } \\
\text { features }\end{array}$} & Features of AIH \\
& Ulcerative colitis \\
& $\begin{array}{l}\text { AMA - } \\
\text { Abnormal cholangiogram or normal cholangiogram (small duct disease) }\end{array}$ \\
\hline Histology & $\begin{array}{l}\text { Cholangitis } \\
\text { Cholestasis }\end{array}$ \\
\hline Treatment & Prednisolone and UDCA \\
\hline
\end{tabular}

Up to $94 \%$ of AlH/PSC overlap patients have antinuclear antibodies, antismooth muscle antibodies, or anti-liverkidney antibodies at titres of $\geq 1: 40$, which is comparable to $\mathrm{AlH}$ and higher than in PSC. ${ }^{7,8}$ Aspartate aminotransferase (AST) level at presentation is lower in AIH/PSC than in AIH, while serum globulins and IgG levels are higher in $\mathrm{AlH} /$ PSC than in PSC. ${ }^{7}$ However, there is significant challenge in making definitive diagnosis of AIH/PSC overlap syndrome because the diagnostic scoring systems used are only validated for $\mathrm{AlH}$ and there is no dedicated diagnostic criteria for AlH/PSC overlap. ${ }^{2,8}$ When assigning a diagnosis of AIH/PSC, experts opine cautious use of the International Autoimmune Hepatitis Group (IAIHG) scoring system in clinical practice and promote the importance of clinical judgement. ${ }^{9}$ Whenever diagnosis of AIH/ PSC overlap is suspected, key diagnostic tools are MRCP and liver histology, and then a final decision along with staging can be made as a combination of these results irrespective of IAIHG score. Care should be taken with regards to the biliary changes that can be seen in $\mathrm{AlH}$, which do not represent a cholangiopathy of PSC. ${ }^{10,11}$

The combination of prednisolone and Azathioprine (AZA), along with low dose ursodeoxycholic acid is a wellestablished first line treatment for patients with $\mathrm{AlH} /$ PSC which was also followed in our patient. Although the majority of patients respond well to this therapy, up to $20 \%$ of patients do not respond. The same incomplete response to azathioprine occurred in our patient as well. The reasons for such treatment failure include drug intolerance or refractory disease. In these patients, second-line drugs like tacrolimus or MMF can be used. Our patient was started on tacrolimus. Most of the adverse events associated with tacrolimus are dose-dependent and correlate with the blood levels of tacrolimus. ${ }^{12}$ Common side effects include tremors, headache, and hyperkalaemia. Less common but major established adverse events include nephrotoxicity, neurotoxicity, and increased risk of neoplasia. Nephrotoxicity due to tacrolimus remains strongly correlated to the administered dose of tacrolimus and the risk is higher at a dose of $0.3 \mathrm{mg} / \mathrm{kg} /$ day compared to a dose of $0.15 \mathrm{mg} / \mathrm{kg}$ twice per day. In several published studies, acute tacrolimus nephrotoxicity has been associated with relatively higher systemic exposure to tacrolimus, although other studies suggest that nephrotoxicity with tacrolimus can occur even at blood levels below the known toxicity threshold. These adverse effects may occur due to a relatively higher starting dose of tacrolimus with respect to the body weight and presence of significant fibrosis on baseline liver biopsy. Targeting low serum levels of tacrolimus ( $<6 \mathrm{ng} /$ $\mathrm{ml}$ ) reduces the risk of such events. In our case also, the patient developed AKI following treatment with tacrolimus. Suspecting tacrolimus associated nephrotoxicity, the drug was tapered and stopped, after which the renal functions improved, giving an indirect evidence of the drug related toxicity. Even though the cholestatic jaundice and AKI improved after treatment with antibiotics indicating them all to be a spectrum of complication of the underlying sepsis, yet the patient was not restarted on tacrolimus in view of its heterogenous behaviour with response to dose-toxicity relationship. Thereafter, the patient was started on MMF.

Mycophenolate Mofetil (MMF) appears to be a highly effective drug for the treatment of patients with autoimmune hepatitis who are unresponsive to azathioprine, or as an alternative where azathioprine is poorly tolerated. It also provides a valuable alternative therapy which can reduce the need for long-term maintenance therapy with corticosteroids. Richardson et al. Showed that 5 of 7 patients with active disease responded to MMF, with a significant improvement in liver histology and good tolerability. ${ }^{13}$ In a study by Jothimani et al., The response to MMF was seen in $74 \%$ (14 patients). MMF treatment failure was observed in $5(26.3 \%)$ patients in whom drug intolerance was noted in three patients. In view of incomplete response to azathioprine and intolerance to tacrolimus, we started our patient on MMF for long term maintenance therapy along with steroids(for the present flare of the disease), after which steroids were gradually tapered off.

\section{Conclusion}

AlH/PSC overlap is a well known entity. However patients presenting with Azathioprine intolerance are difficult to treat as a subgroup. The next drug tacrolimus has good 
therapeutic efficacy but its nephrotoxicity needs to be considered always.

In this case urosepsis with deranged liver enzymes with a substrate of drug resistant autoimmune hepatitis presented to us a clinical dichotomy-to aggressively manage sepsis and rule out immediate progression of the underlying disease. However, the management of both the conditions is largely different. Major single stricture would have needed ERCP based therapy and multiple progressive strictures would have needed liver transplantation. However, improvement of sepsis, settling down of transaminitis as well as bilirubin, improved blood counts and finally a fresh MRCP finally saved the day. The patient continued to improve, MMF was restarted and the need for urgent transplant could be averted. The clinical differentiation, medical versus GI intervention and relative drug toxicities should always be considered.

\section{Conflict of Interest: None}

\section{References}

1. Carbone M, Neuberger JM. Autoimmune liver disease, autoimmunity and liver transplantation. J Hepatol 2014; 60(1): 210-223.

2. Manns MP, Czaja AJ, Gorham JD, Krawitt EL, MieliVergani G, Vergani D et al. Diagnosis and management of autoimmune hepatitis. Hepatol Baltim Md 2010; 51(6): 2193-2213.

3. Hübener $\mathrm{S}, \mathrm{Oo} \mathrm{YH}$, Than NN, Hübener $\mathrm{P}$, Weiler-Normann C, Lohse AW et al. Efficacy of 6-Mercaptopurine as Second-Line Treatment for Patients With Autoimmune Hepatitis and Azathioprine Intolerance. Clin Gastroenterol Hepatol 2016; 14(3): 445-453.

4. Montano-Loza AJ, Carpenter HA, Czaja AJ. Features associated with treatment failure in type 1 autoimmune hepatitis and predictive value of the model of endstage liver disease. Hepatol Baltim Md 2007; 46(4): 1138-1145.

5. Chapman R, Fevery J, Kalloo A, Nagorney DM, Boberg KM, Shneider B et al. American Association for the Study of Liver Diseases. Diagnosis and management of primary sclerosing cholangitis. Hepatol 2010; 51(2): 660-678.

6. Czaja AJ. Frequency and nature of the variant syndromes of autoimmune liver disease. Hepatol 1998; 28(2): 360-365.

7. Kaya M, Angulo P, Lindor KD. Overlap of autoimmune hepatitis and primary sclerosing cholangitis: an evaluation of a modified scoring system. J Hepatol 2000; 33(4): 537-542.

8. Yeoman AD, Westbrook RH, Al-Chalabi T, Carey I, Heaton ND, Portmann BC et al. Diagnostic value and utility of the simplified International Autoimmune Hepatitis Group (IAIHG) criteria in acute and chronic liver disease.
Hepatol 2009; 50(2): 538-545.

9. Chazouillères $O$. Diagnosis of primary sclerosing cholangitis--autoimmune hepatitis overlap syndrome: to score or not to score? J Hepatol 2000; 33(4): 661-663.

10. Czaja AJ, Carpenter HA. Autoimmune hepatitis with incidental histologic features of bile duct injury. Hepatol 2001; 34(4 Pt 1): 659-665.

11. Lewin $M$, Vilgrain V, Ozenne $V$, Lemoine $M$, Wendum $\mathrm{D}$, Paradis $\mathrm{V}$ et al. Prevalence of sclerosing cholangitis in adults with autoimmune hepatitis: a prospective magnetic resonance imaging and histological study. Hepatol 2009; 50(2): 528-537.

12. Yagihashi A, Yoshida Y, Terasawa K, Ujike Y, Konno A, Ogura K et al. The effect of FK 506 on peripheral blood T-lymphocyte subsets in orthotopic liver transplant patients. Transplant Proc 1991; 23(6): 2937-2938.

13. Richardson PD, James PD, Ryder SD. Mycophenolate mofetil for maintenance of remission in autoimmune hepatitis in patients resistant to or intolerant of azathioprine. J Hepatol 2000; 33(3): 371-375.

14. Jothimani D, Cramp ME, Cross TJ. Role of mycophenolate mofetil for the treatment of autoimmune hepatitis-an observational study. J Clin Exp Hepatol 2014; 4(3): 221-225. 